\title{
La percepción del espacio escolar y su impacto en el aprendizaje de estudiantes de secundaria a profesional en CDMX
}

\author{
The Perception of School Space and its Impact on the Learning of Secondary \\ Students to Professionals in CDMX
}

\section{A percepção do espaço escolar e seu impacto na aprendizagem de alunos do ensino médio ao profissional no CDMX}

\author{
Gerardo Chávez García \\ Universidad del Distrito Federal, Campus Santa María, México \\ architecti_mex@yahoo.com.mx \\ https://orcid.org/0000-0002-0307-5513
}

\section{Resumen}

El resultado del presente trabajo muestra los hallazgos encontrados respecto a la percepción que tienen algunos estudiantes sobre los espacios donde aprenden o practican algún deporte. Entre ellos el hecho de que son necesarias las mejoras de las condiciones espaciales para coadyuvar en el aprendizaje y la superación personal. El objetivo de esta investigación realizada en Ciudad de México a principios del año 2019 fue evaluar y entender las circunstancias que se viven en la actualidad en el espacio escolar, tan importante para el progreso y la competitividad en el país. La investigación se realizó dentro del paradigma hermenéutico interpretativo; es de corte cualitativo. Se realizaron dos entrevistas en estudiantes becados de secundaria y preparatoria, más un grupo de enfoque de nivel profesional, con beca deportiva, como informantes clave de la propia comunidad de estudio. Asimismo, se reunió y exploró material teórico, descriptivo de diversos autores de diferentes épocas para la comprensión cualitativa crítico-analítica, dentro del paradigma de la complejidad, gracias a lo cual se generó un modelo de análisis para la comprensión de 


\section{Revista Iberoamericana \\ de las Ciencias Sociales y \\ Humanísticas}

ISSN: $2395-7972$

la información y actuación con miras a la mejora de los espacios educativos. Finalmente, se arribó a la conclusión de que hay un desequilibrio de las condiciones y equipamiento de escuelas para las actividades académicas, con gran demanda en México ante un mundo altamente competitivo.

Palabras clave: impacto en el aprendizaje, instalación educativa, percepción.

\section{Abstract}

The result of this work shows the findings found regarding the perception that some students have about the spaces where they learn or practice a sport. Among them the fact that improvements in spatial conditions are necessary to contribute to learning and selfimprovement. The objective of this research conducted in Mexico City at the beginning of 2019 was to evaluate and understand the circumstances that are currently lived in the school space, being so important for progress and competitiveness in the country. The research was conducted within the interpretive hermeneutic paradigm. It is of qualitative nature. Two interviews were conducted in middle school and high school scholarship students, plus a professional level focus group, with sports scholarship, as key informants of the study community itself. Likewise, theoretical and descriptive material from various authors from different eras was collected and explored for qualitative critical-analytical understanding, within the complexity paradigm, thanks to which an analysis model was generated for the understanding of information and action to the improvement of educational spaces. Finally, it was concluded that an imbalance of the conditions and equipment of schools for academic activities, with great demand in our country before a highly competitive world.

Keywords: impact on learning, educational installation, perception.

\section{Resumo}

O resultado deste trabalho mostra os achados encontrados sobre a percepção que alguns alunos têm sobre os espaços onde aprendem ou praticam um esporte. Entre eles, o fato de que são necessárias melhorias nas condições espaciais para contribuir com o aprendizado e o auto-aperfeiçoamento. O objetivo desta pesquisa realizada na Cidade do México, no início do ano de 2019, foi avaliar e entender as circunstâncias atualmente vividas no espaço escolar, tão importantes para o progresso e a competitividade no país. A pesquisa foi 


\section{Revista Iberoamericana \\ de las Ciencias Sociales y \\ Humanísticas}

ISSN: $2395-7972$

realizada dentro do paradigma hermenêutico interpretativo; É qualitativo. Duas entrevistas foram realizadas em estudantes do ensino médio e do ensino médio, além de um grupo focal de nível profissional, com uma bolsa esportiva, como informantes-chave da própria comunidade de estudo. Da mesma forma, materiais teóricos e descritivos de diferentes autores de diferentes épocas foram coletados e explorados para o entendimento qualitativo crítico-analítico, dentro do paradigma da complexidade, graças ao qual foi gerado um modelo de análise para a compreensão das informações e ações com Você procura a melhoria dos espaços educacionais. Por fim, concluiu-se que há um desequilíbrio nas condições e equipamentos das escolas para atividades acadêmicas, com grande demanda no México em um mundo altamente competitivo.

Palavras-chave: impacto na aprendizagem, instalação educacional, percepção.

Fecha Recepción: Mayo 2019

Fecha Aceptación: Diciembre 2019

\section{Introducción}

Ante las necesidades de un mundo globalizado y sumamente competitivo, la problemática de las acciones políticas poco coherentes dentro del sistema educativo de México, la deserción escolar, el bajo rendimiento académico y en el deporte, aparecen múltiples factores para el aprendizaje y el compromiso que influyen en la formación de estudiantes y deportistas y profesionistas. Esta investigación se orienta únicamente a los espacios educativos con el objetivo de ampliar el conocimiento respecto a la forma en que los educandos perciben a estos para su desarrollo. Y en esa línea se encontraron respuestas más allá del sentido pedagógico, en el ejercicio para la formación educativa. Este trabajo analizó las opciones para promover ciertas competencias y mecanismos que justifican la necesidad de incorporar escuelas de calidad, las cuales son necesarias para la motivación de sujetos participantes, con la asunción de un compromiso de mejora continua.

De manera más específica, el objeto del presente estudio fue conocer la evidencia de las condiciones y percepciones que los estudiantes manifiestan sobre los espacios como medios donde se desarrollan de manera integral. Así, se realizó una investigación cualitativa con base en dos instrumentos: dos encuestas más un grupo de enfoque compuesto de informantes seleccionados acorde a su rendimiento académico y deportivo, así como también la revisión y análisis de la literatura considerada para este importante 


\section{Revista Iberoamericana \\ de las Ciencias Sociales y \\ Humanísticas}

ISSN: $2395-7972$

tema. Las entrevistas se realizaron para evaluar si el aprendizaje se potencializa cuando es realizado dentro de espacios de confort y buenas instalaciones de la escuela o colegio, al igual que para identificar las expectativas y necesidades que tienen los estudiantes. Consecuentemente, luego de un contraste con la literatura seleccionada, se estableció un modelo final con varias brechas posibles de actuación y solución a la pregunta de investigación. Como parte de los resultados se encontró que entre mejor sea la capacidad instalada en una escuela, mayor será el compromiso de aprendizaje y rendimiento deportivo.

\section{Materiales y método}

El tipo de estudio es exploratorio, descriptivo, relacional y empírico; y pertenece a un fenómeno contemporáneo dentro del paradigma hermenéutico interpretativo. La investigación se realizó dentro de un proceso de búsqueda con la finalidad de llegar a una explicación y comprensión de una realidad específica, delimitada por un tiempo y un contexto, con parámetros del paradigma de la investigación cualitativa, fundamentándose en el proceso de comprensión cualitativa, analizando aspectos teóricos e instrumentos de investigación con base en entrevistas más un grupo de enfoque para entender el aspecto de la percepción del bienestar. Ante todo, analizar las condiciones que los individuos perciben respecto al ambiente; entender cómo puede integrarse adquiriendo un compromiso de superación y reafirmación axiológica en un mundo competitivo. Y estudiar de forma crítica parte de las teorías con las condiciones actuales de los espacios de la organización escolar con informantes claves de la propia comunidad de estudio con la finalidad de llegar a una explicación y comprensión de una realidad específica, delimitada por un tiempo y un contexto, como ya se mencionó.

\section{Método de muestreo}

Se utilizó un muestreo no probabilístico. La muestra fue representativa. Se realizaron dos entrevistas a estudiantes seleccionados de secundaria y preparatoria, más un grupo de enfoque de nivel profesional. Además, se reunió y analizó material teórico, de diversos autores, dentro del paradigma de la complejidad. 


\section{Revista Iberoamericana \\ de las Ciencias Sociales y \\ Humanísticas}

ISSN: $2395-7972$

Con el muestreo opinático la realización del trabajo de campo se simplifica pues se puede concentrar mucho la muestra. Sin embargo, al querer concentrar la muestra, se pueden cometer errores y sesgos debidos al investigador y, al tratarse de un muestreo subjetivo (según las preferencias del investigador), los resultados de la encuesta no tienen una fiabilidad estadística exacta (Conceptos básicos de la metodología de la investigación, 2010, párr. 20).

Tabla 1. Variables de estudio e indicadores

\begin{tabular}{|l|l|l|l|}
\hline $\begin{array}{l}\text { Variable } \\
\text { cualitativa }\end{array}$ & \multicolumn{1}{|c|}{ Del concepto } & Operacional & Indicadores \\
\hline - Espacio & "El espacio educativo como & - Estética & - Percepción del \\
educativo & memoria, transformación y & - Instalaciones & bienestar \\
- & $\begin{array}{l}\text { crecimiento, que recoja los } \\
\text { Aprendizaje }\end{array}$ & - Docentes & - Actitud para ser mejor \\
& $\begin{array}{l}\text { procesos temporales y de vida de } \\
\text { (Abad, 2006, p. 2) }\end{array}$ & - Aula & - Comodidad \\
& & & \\
\hline
\end{tabular}

Fuentes: Elaboración propia

La presente investigación analizó si el espacio educativo en las aulas y espacios escolares de Ciudad de México motivan la actitud para la enseñanza aprendizaje.

\section{Marco teórico}

En esta sección se mencionan algunas de las citas más importantes para este tema de investigación.

Cualquier cambio que se pretenda llevar a cabo en el sistema educativo puede ir al traste si no se adecuan los espacios donde se han de llevar a cabo. (p. 1) Es necesario pasar de una etapa cuantitativa de satisfacción de puestos escolares a una etapa cualitativa en la que la arquitectura escolar, como medio ambiente físico, aporte y facilite calidad a la educación" (Salmerón, 1989). 


\section{Revista Iberoamericana \\ de las Ciencias Sociales y \\ Humanísticas}

ISSN: $2395-7972$

Este trabajo precisamente se refiere a la vertiente cualitativa. ¿Cómo mejorar el aprendizaje de los estudiantes? A través de los espacios educativos. La información teórica recolectada para esta investigación se encuentra en proceso de consolidación investigativa, mediante la búsqueda de códigos o categorías que se siguen construyendo e indagando y que son de sustancial actividad para la fundamentación teórica.

Según Casassus (1992), en la actualidad mexicana está presente la modernización educativa y gran parte corresponde al sector gubernamental por medio de la Secretaría de Educación Pública (SEP), sin darle importancia a los espacios educativos. Modernidad educacional y modernización educativa son las condiciones para lograr, por una parte, lo que se espera: "educar a nuevas generaciones; y por otra, (...) una buena relación entre el mejor uso de los recursos humanos, físicos y financieros a su disposición y la calidad del resultado del proceso de enseñanza-aprendizaje" (Casassus, 1992, p. 39).

El objetivo de la planeación arquitectónica es, siguiendo esta vez a Coppola (1997), satisfacer las exigencias espaciales del hombre, término que indica "además de las exigencias biológicas-funcionales, también las psicológicas, es decir, las exigencias ligadas al comportamiento, al modo de uso del espacio, al significado simbólico del espacio mismo (p. 33).

Figura 1. Vistas exteriores de escuelas en las colonias Juárez y Roma.

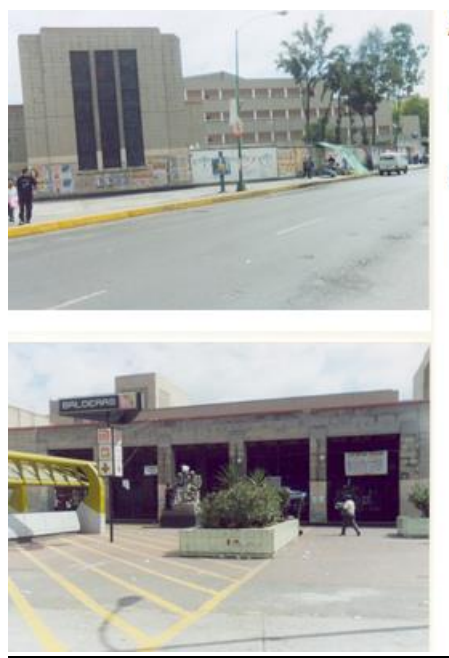

A la Izquierda Escuela primaria

Ubicada en Avenida Chapultepec

Esquina con Balderas en el centro

De la ciudad de México. (24)

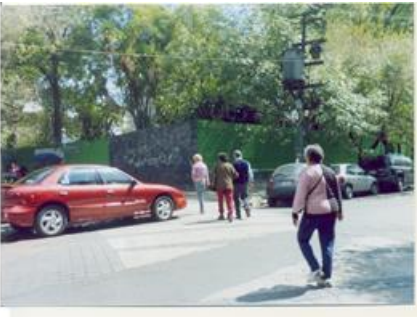

Lado derecho imagen de la

Fachada exterior de la primaria

Entre las calles de Medellín y

Medellín y Colima, CDMX

Foto (25)

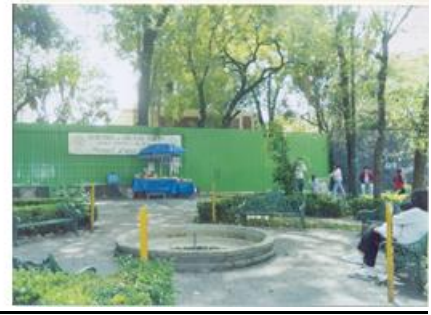

Fuente: Elaboración propia

El análisis del estado del arte refleja que las escuelas o institutos son también un espacio organizacional y se requiere de sus mejoras para el rendimiento estudiantil. En esa línea, Ortigosa et al. (2003) se propusieron "conocer las características de la prosecución y 


\section{Revista Iberoamericana \\ de las Ciencias Sociales y \\ Humanísticas}

ISSN: $2395-7972$

el rendimiento estudiantil, a través de la evaluación del pensum 2000, tomando como elementos clave, el rendimiento y la prosecución del estudiante a lo largo de su carrera académica" (párr. 1).

Por otro lado, un colegio de prestigio llamado Reggio Emilia ha buscado lo siguiente:

Un diálogo amable entre arquitectura, diseño y pedagogía para proyectar unas escuelas bellas, sugerentes en posibilidades e innovadoras. En definitiva: una concepción del espacio educativo como memoria, transformación y crecimiento, que recoja los procesos temporales y de vida de toda la comunidad educativa (Abad, 2006, p. 2).

Y en la misma fuente se citan, a modo de epígrafe, las palabras de Loris Malaguzzi, quien considera que el aula infantil "debiera ser una especie de acuario transparente donde se reflejaran las ideas, las actitudes y las personas" (citado en Abad, 2006, p. 1).

Cualquier actividad humana necesita un espacio y tiempo determinados. "Así sucede con el enseñar y el aprender, con la educación. Resulta de eso que la educación posee una dimensión espacial y que, también, el espacio sea, junto con el tiempo, un elemento básico, constitutivo, de la actividad educativa" (Frago, 1998, citado en Abad, 2006, p. 3).

Las citas de las investigaciones anteriores demuestran la importancia del espacio educativo. Como podemos observar, existen diferentes enfoques necesarios para avanzar en lo que hacemos. Las organizaciones, el rol administrativo, la planeación, el sistema educativo demuestran los múltiples factores que existen para provocar las mejoras en el rendimiento y bienestar estudiantil.

Quizás por ello se evidencia, en la revisión bibliografía sobre el tema, la diversidad de puntos de vista y de factores a través de los cuales ha sido estudiado el problema del rendimiento. Lo que sí se ha demostrado es que en el rendimiento estudiantil intervienen múltiples factores tanto individuales como institucionales y socio-económicos, por lo que si bien es cierto que la cuantificación de sus indicadores es necesaria para caracterizar y diagnosticar o describir la situación actual, no considerar los aspectos cualitativos del mismo hace que los resultados del estudio sean insuficientes 


\section{Revista Iberoamericana \\ de las Ciencias Sociales y \\ Humanísticas}

ISSN: $2395-7972$

para la toma de decisiones en la aplicación de correctivos para mejorar el problema del rendimiento (Ortigosa et al., 2003, párr. 17).

Este trabajo recurrió al paradigma de la complejidad. De acuerdo con Morín (2008, citado en Logroño, 2014), "la complejidad es la incertidumbre en el seno de los sistemas ricamente organizados" (p. 1). Además, es parte, subyace dentro del paradigma de la simplicidad. Este último "pone un orden en el universo, y persigue el desorden. El orden se reduce a una ley, a un principio" (Pernia, s. f., p. 1).

Ahora bien, sobre la toma de decisiones vale la pena retomar el siguiente fragmento:

No cabe duda alguna que la ciencia de la psicología puede (y debe) jugar un rol en todo proyecto arquitectónico, por cuanto el arquitecto y/o diseñador están en grado de crear los diversos ambientes que pueden influir en los estados de ánimo de los moradores de estos espacios, sin importar si estos están destinados a intereses privados, públicos o institucionales

Muchas veces se diseña alguna estructura sin tomar en consideración las “emociones", "las características de personalidad", "el tipo de cultura de origen" (Goleman, 2003), etc., de quienes posteriormente ocuparán las dependencias ocupadas (Lotito, 2009, p. 14).

En diversos artículos analizados se hace mención de los sentidos, mirar al futuro, la humanidad.

Figura 2. Tendencias y códigos más representativos obtenidos del marco teórico
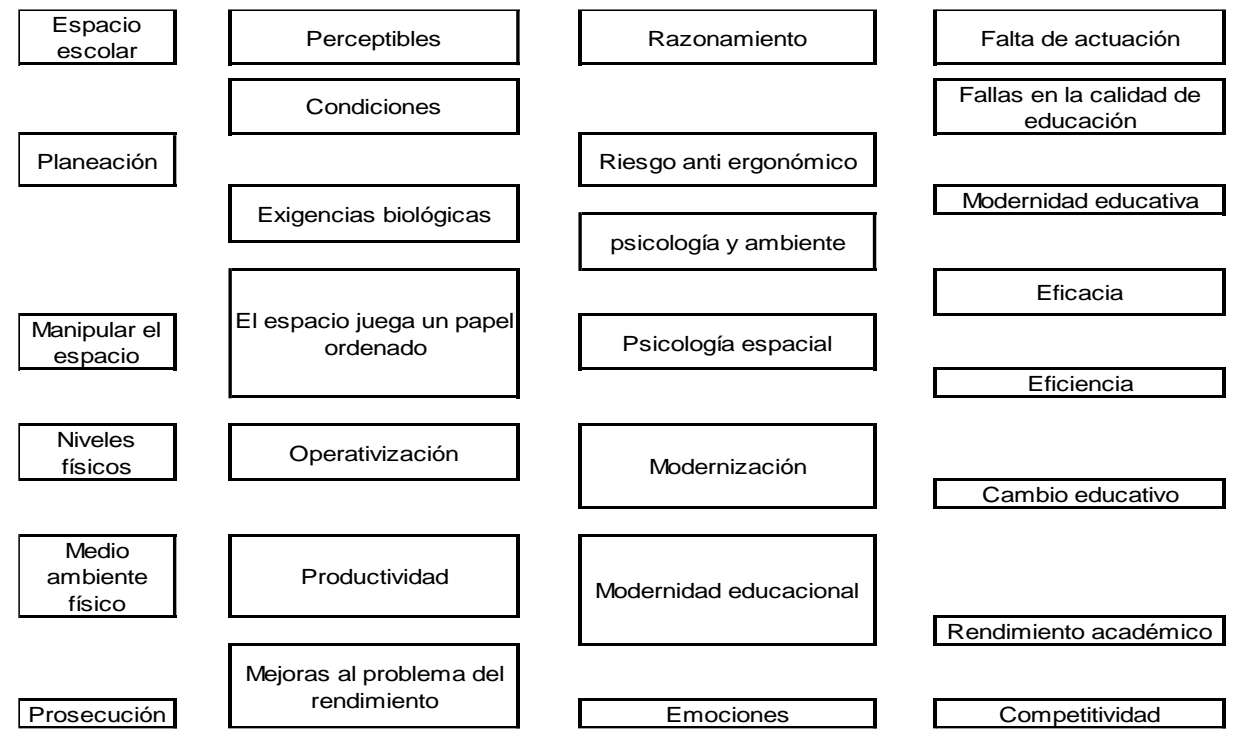

Fuente: Elaboración propia 


\section{Revista Iberoamericana \\ de las Ciencias Sociales y \\ Humanísticas}

ISSN: $2395-7972$

Los resultados del análisis del marco teórico visualizan la necesidad de mejorar la calidad de la educación. Para el caso que nos ocupa, la problemática que persiste es la persistencia de no aplicar la teoría para las mejoras en la educación.

Figura 3. Trabajo de campo: actividades en el exterior de una escuela primaria

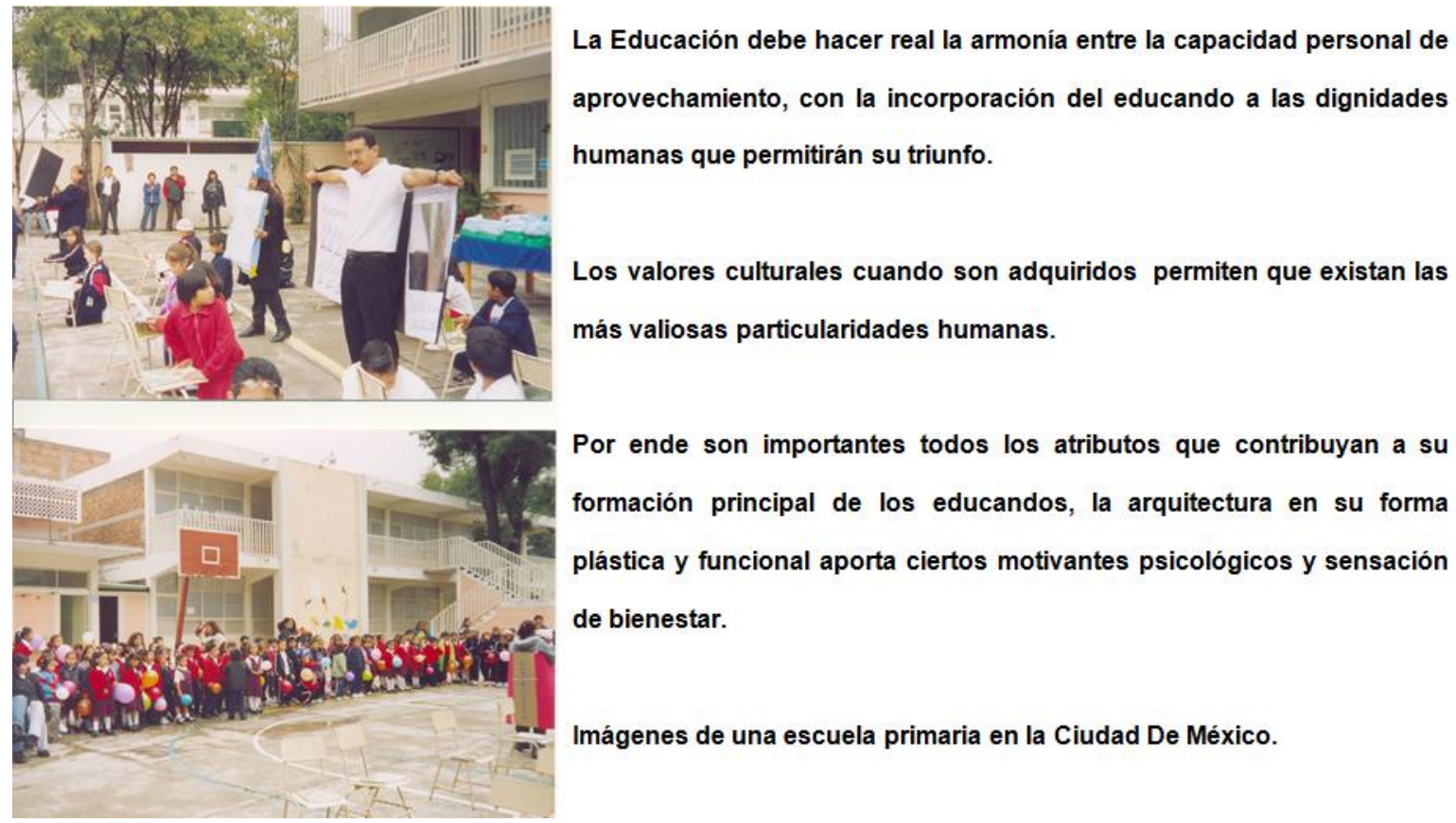

Fuente: Elaboración propia

"La característica más importante de un sistema autopoiético es que se levanta sobre sus propias condiciones y se constituye como distinto del mundo circundante a través de su propia dinámica, de tal manera que ambas cosas sean inseparables" (Maturana, citado en López, 1998, p. 112).

Sin embargo:

No entendamos esta resistencia al modo exasperado de gran parte de las ideologías de nuestro tiempo, pues "sonreír, reír, bromear, jugar, acariciar, abrazar, es también resistir".

(...) "Resistir, resistir primero a nosotros mismos, a nuestra indiferencia y nuestra falta de atención, nuestro cansancio y nuestro desaliento, nuestros malos impulsos y nuestras mezquinas obsesiones" (López, 1998, p. 113).

Estos últimos antecedentes teóricos fundamentan la presente investigación. 


\section{Revista Iberoamericana \\ de las Ciencias Sociales y \\ Humanísticas}

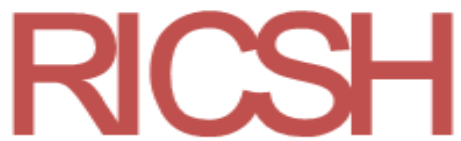

ISSN: 2395 - 7972

Figura 4. Escuela primaria rural al oriente de Ciudad de México

\begin{abstract}
El presente trabajo investigativo reúne un sustento para promover la mejora en estos espacios educativos, causar la calidad en sus instalaciones, debe ser una prioridad.

En estas imágenes se observan las carencias de instalaciones en poblados no tan lejanos en la periferia de la Ciudad De México y que merecen escuchados también.

La capacidad instalada para la enseñanza y aprendizaje es muy escasa
\end{abstract}

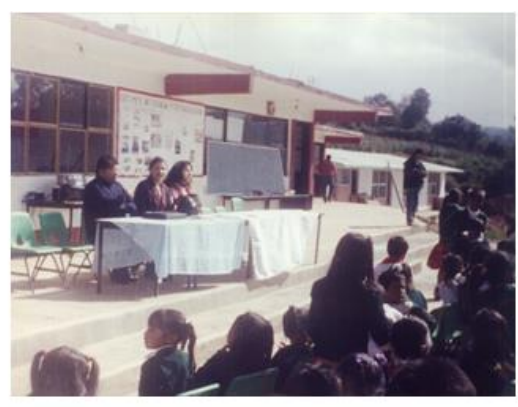

32.- Personal docente y administrativo

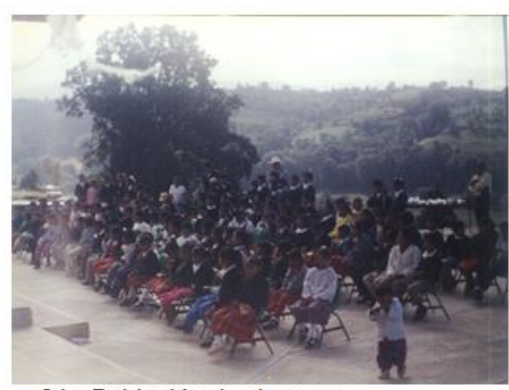

34 - Población de alumnos

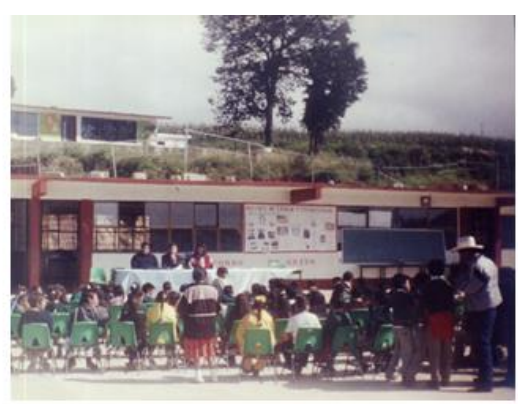

33.- Padres de familia

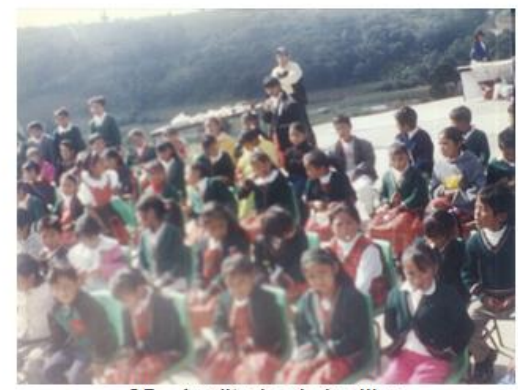

35. Auditorio al aire libre

Fuente: Elaboración propia

\section{Datos de las entrevistas y grupo de enfoque}

\section{Entrevista uno}

Se realizaron encuestas y un grupo de enfoque. Respecto a las primeras, a manera de ejemplo se muestra lo siguiente:

- Hola, ¿cómo estás? [Romper el hielo].

- ¿Cómo te llamas? ¿Cuántos años tienes? ¿Dónde estudias?

- $\quad$ Es un gusto conocerte. 


\section{Revista Iberoamericana \\ de las Ciencias Sociales y \\ Humanísticas}

ISSN: $2395-7972$

Figura 5. Proceso de la entrevista

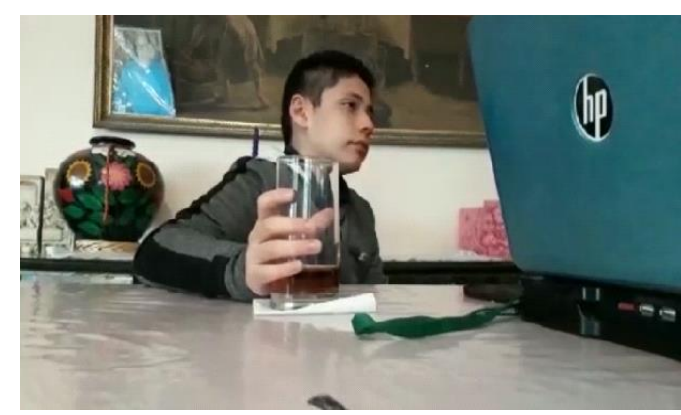

Fuente: Elaboración propia

Figura 6. Proceso de la entrevista

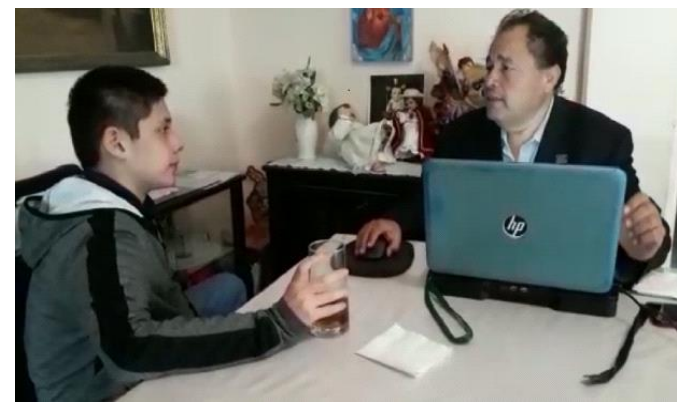

Fuente: Elaboración propia

- ¿QQué opinas de tu escuela? R = Mi escuela pública es de las más reconocidas del Distrito Federal, recomendada por diferentes amistades. Me siento orgulloso de estar ahí.

- $\quad$ ¿Cómo percibes los espacios de tu escuela? $\mathrm{R}=$ Considerablemente grandes comparados con la primaria donde estudié.

- $\quad$ Para ti, Emmanuel, ¿cuál es el área o espacio más importante? $\mathrm{R}=\mathrm{El}$ patio central tiene una fuente rodeada por muchos árboles. Es un lugar muy hermoso, van muchos pajaritos y es agradable estar ahí.

- Por favor, habla de tu experiencia en el espacio educativo. $\mathrm{R}=$ Como lo había dicho, en la escuela primaria era muy pequeña. En esta escuela secundaria es más cálida. Es mucho más grande y la siento más cómoda. Aunque el director es más estricto, es más cálida. El mobiliario era más cómodo, el de la primaria, y el mobiliario de esta secundaria es muy incómodo, aunque la luz, la ventilación, las ventanas, iluminación y el calor es bueno. 


\section{Revista Iberoamericana \\ de las Ciencias Sociales y \\ Humanísticas}

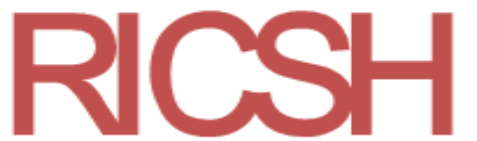

ISSN: $2395-7972$

- ¿Qué disfrutas más de tus estudios? $\mathrm{R}$ = Lograr ganar algunos concursos y mantener mi beca.

- ¿QQué es lo que te atrae más de tu escuela? $\mathrm{R}=$ Me atrae pensar que es de las mejores escuelas y me siento orgulloso de pertenecer a este plantel.

- ¿Qué entiendes por el espacio educativo? $\mathrm{R}=\mathrm{El}$ espacio educativo es importante para aprender mejor, pues si el espacio es más cómodo vas a aprender mejor, porque cuando el espacio es incómodo solo te concentras en la incomodidad del espacio y no tanto en lo que estás aprendiendo.

- Tus principales motivaciones para estudiar. $\mathrm{R}=$ Es convivir o estudiar con las personas más cercanas, y me siento cómodo que la escuela sea más grande, sentirme orgulloso de la escuela aunque no estudie mucho.

- ¿Con qué personas te gusta trabajar? $\mathrm{R}=$ Con mis amigos más cercanos.

- ¿ ¿Cuál es tu mayor reto actualmente? $\mathrm{R}=$ Mantener la calificación de 8.5 para mantener mi beca.

- $\quad$ ¿Te gusta estudiar? $\mathrm{R}=\mathrm{A}$ veces. Me molesta no entender algunas cosas o que no las expliquen bien.

- $\quad$ Platícame tu experiencia. ¿Cómo disfrutas los espacios, las instalaciones de las áreas y hoteles que visitas? $\mathrm{R}=\mathrm{El}$ espacio no cambia mucho, tiene una mesa, una silla, lo que llama la atención es al exterior, ya que tienen áreas muy grandes, se percibe un ambiente más tranquilo, más cómodo y se disfruta del bosque.

Con el software Atlas.ti se definieron los siguientes códigos para el análisis de la entrevista uno. 


\section{Revista Iberoamericana de las Ciencias Sociales y Humanísticas}

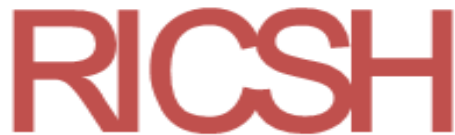

Figura 7. Definición de códigos de la entrevista uno

[1:5] primaria era echo muy
pequeña
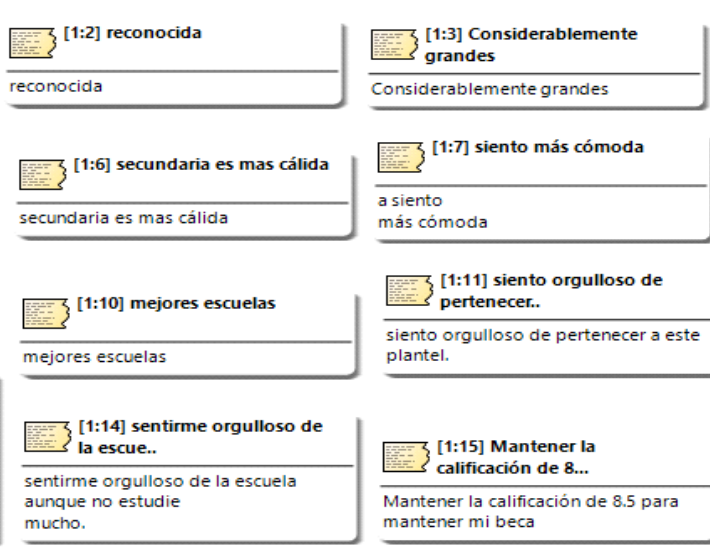

[1:4] El patio central, tiene

El patio central, tiene una fuente rodeada por muchos árboles, es un pajaritos yes agradable

Fuente: Elaboración propia

\section{Entrevista dos}

Figura 8. Proceso de la entrevista

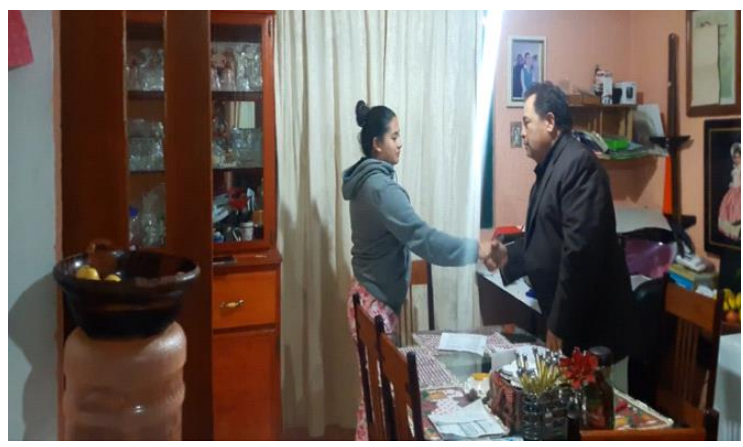

Fuente: Elaboración propia

FE] $[1: 8]$ la luz, la ventilación, las 3 ve. la luz, la ventilación, las ventanas y el colar controlado es bueno

[1:12] El espacio educativo es

El espacio educativo es importante para aprender

siel espacio es más

cómodo vas a aprender mejor porque

cuando el espacio es incómodo solo

te concentras en la incomodidad de

aprendiendo. 


\section{Revista Iberoamericana \\ de las Ciencias Sociales y \\ Humanísticas}

Figura 9. Proceso de la entrevista

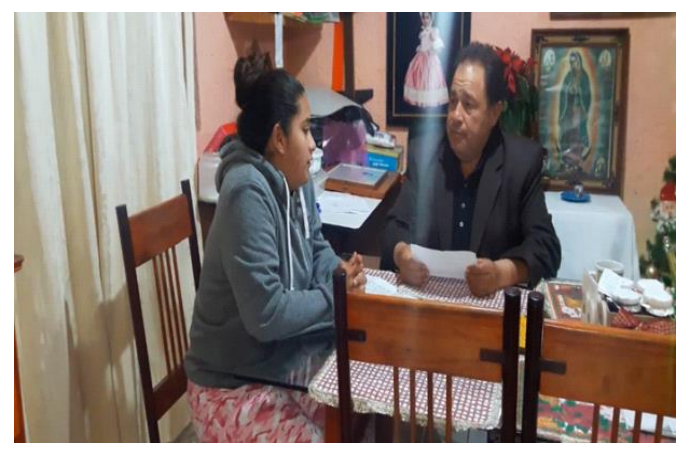

Fuente: Elaboración propia

Al igual que en el caso anterior, con el software Atlas.ti se definieron los siguientes códigos para el análisis de la entrevista dos.

Figura 10. Definición de códigos de la entrevista dos
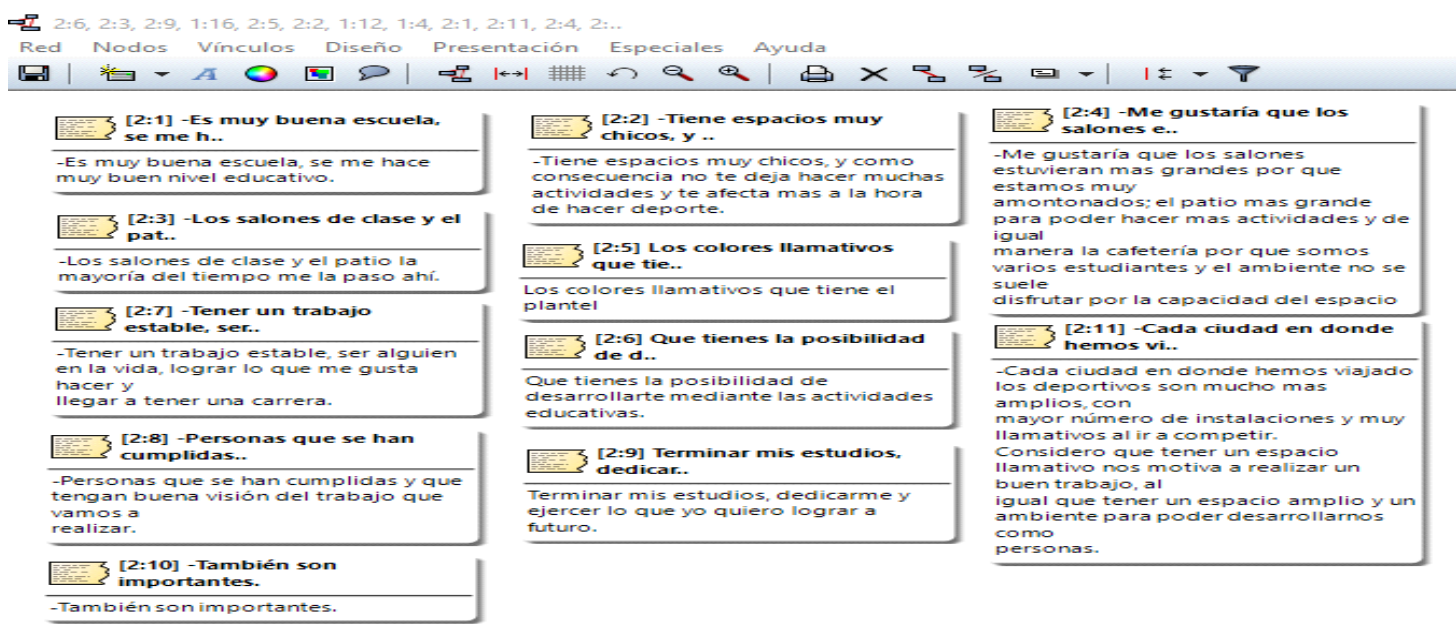

Fuente: Elaboración propia

\section{Grupo de enfoque}

Se preparó una breve presentación de un libro de espacios deportivos. Posteriormente se llevó a cabo el grupo de enfoque. 
Revista Iberoamericana

de las Ciencias Sociales y

Humanísticas

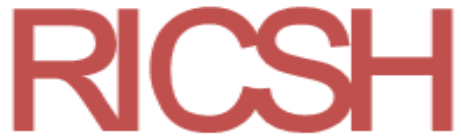

ISSN: $2395-7972$

Figura 11. Grupo de enfoque

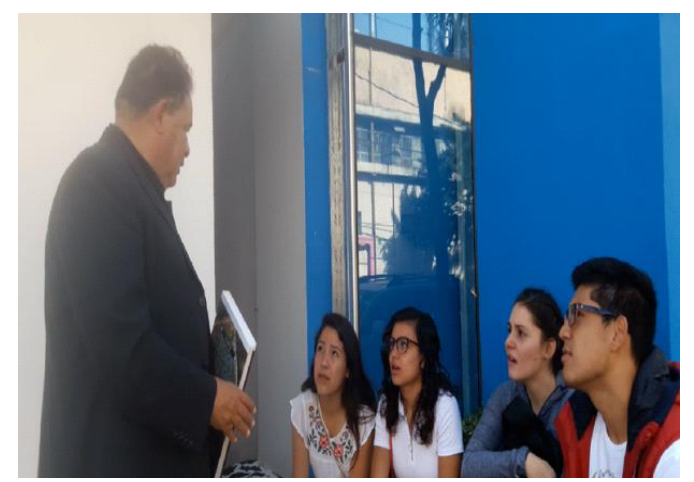

Fuente: Elaboración propia

Figura 12. Grupo de enfoque

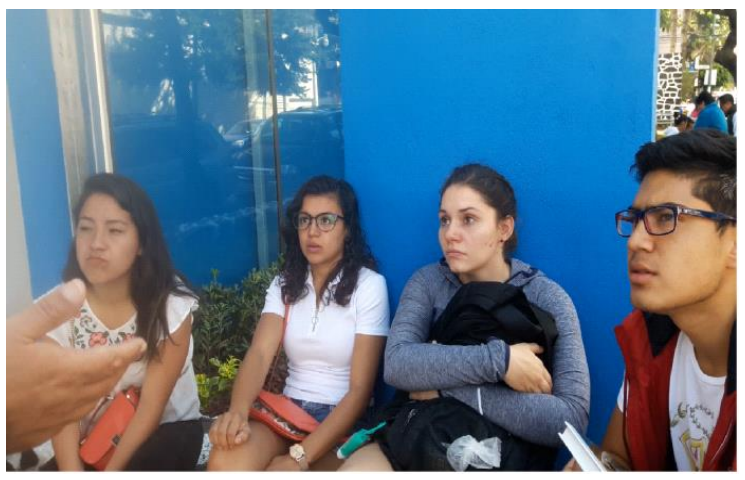

Fuente: Elaboración propia

Esto fue realizado para poder interpretar y entender los espacios donde trabajan, las instalaciones de los planteles de sus escuelas. 


\section{Revista Iberoamericana de las Ciencias Sociales y Humanísticas}

Figura 13. Red Atlas.ti. Definición de códigos del grupo de enfoque

댐 $3: 3,3: 9,3: 6,3: 16,3: 2,3: 5,3: 15,3: 12,3: 4,3: 11,3: 14$, ..

Red Nodos Vínculos Diseño Presentación Especiales Ayuda

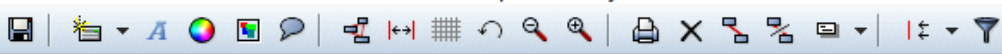

\begin{tabular}{|c|c|}
\hline 壏 $\left\{\begin{array}{l}{[3: 1]=\text { Luis: Opino que va a }} \\
\text { definir .. }\end{array}\right.$ & $\begin{array}{l}\text { [3:2] -Cristina: Bueno a mi } \\
\text { parecer .. }\end{array}$ \\
\hline $\begin{array}{l}\text { = Luis: Opino que va a definir la } \\
\text { preparación y la forma en como nos } \\
\text { desenvolvamos. }\end{array}$ & \multirow{3}{*}{$\begin{array}{l}\text {-Cristina: Bueno a mi parecer estas } \\
\text { invirtiendo en una educación integral, } \\
\text { como podría ser el deporte te está } \\
\text { aportando a los estudiantes disciplina } \\
\text { y el problema es que lo fomentan más } \\
\text { académicamente pero como no lo } \\
\text { toman mucha importancia. }\end{array}$} \\
\hline $\begin{array}{l}\text { [3:5] Si, por que yo estudio } \\
\text { fisiote.. }\end{array}$ & \\
\hline \multirow{3}{*}{$\begin{array}{l}\text { Si, por que yo estudio fisioterapia y } \\
\text { en mi universidad si hay apoyo en } \\
\text { laboratorios y materiales, pero en } \\
\text { cuanto a instalaciones deportivas son } \\
\text { muy escasas, considero que tendrían } \\
\text { que haber mejores instalaciones. }\end{array}$} & \\
\hline & $\begin{array}{l}{[3: 6] \text { Est }} \\
\text { segundo }\end{array}$ \\
\hline & \multirow{3}{*}{$\begin{array}{l}\text { Estoy estudiando el segundo semestre } \\
\text { en UVM centro y considero que en lo } \\
\text { particular no me agrada tanto porque } \\
\text { en las instalaciones son muy pequeñas } \\
\text { y sus edificaciones son muy viejas, } \\
\text { dicen que aguanta terremotos, pero } \\
\text { yo me siento seguro. }\end{array}$} \\
\hline $\begin{array}{l}{[3: 10] \text { Pues estudie en prepa }} \\
\text { UNAM, si.. }\end{array}$ & \\
\hline \multirow{3}{*}{$\begin{array}{l}\text { Pues estudie en prepa UNAM, si tenía } \\
\text { instalaciones, pero las restringían, se } \\
\text { tiene que complementar las } \\
\text { instalaciones educativas con las } \\
\text { deportivas. Por ejemplo: Mi amiga } \\
\text { Paola va en la Anáhuac y entro con } \\
\text { beca deportiva, pero en su planten no } \\
\text { tienen espacios deportivos, no puedes } \\
\text { entrar a una universidad que te ofrece } \\
\text { algo cuando en realidad no te lo dan. }\end{array}$} & \\
\hline & $\begin{array}{l}\text { [3:11] Por ejemplo, Cuidad } \\
\text { Universita.. }\end{array}$ \\
\hline & $\begin{array}{l}\text { Por ejemplo, Cuidad Universitaria } \\
\text { tiene todos los espacios deportivos y } \\
\text { educativos, pero no son espacios } \\
\text { cerrados y afectan al desarrollo } \\
\text { deportivo. }\end{array}$ \\
\hline $\begin{array}{l}\text { [3:14] No pueden hacer } \\
\text { nuevos espacio.. }\end{array}$ & $\begin{array}{l}\text { [3:13] Pienso que en lu } \\
\text { públicos.. }\end{array}$ \\
\hline $\begin{array}{l}\text { No pueden hacer nuevos espacios } \\
\text { porque no tienen una buena } \\
\text { planeación de las instalaciones en las } \\
\text { universidades. }\end{array}$ & $\begin{array}{l}\text { Pienso que en lugares públicos } \\
\text { porque tenemos que dejar de ver los } \\
\text { intereses propios y ver intereses } \\
\text { comunes. }\end{array}$ \\
\hline
\end{tabular}

i veo la diferencia porque en mi
secundaria si tenia canchas y en mi
universidad no tengo un espacio en
donde pueda hacer deportes, pero en
mi universidad tengo cafetería, pero
en mi secundaria no la tenía, entonces
no hay un balance en el apoyo de
instalaciones en las escuelas.
[3:7] En nuestra escuela solo
nos ap..

En nuestra escuela solo nos apoyaron con un aro de basquetbol y mi coach se estuvo "peleando" con el coordinador de deportes para que nos pusieran un espacio definitivo de deportes, pero no se pudo por que el gobierno les tiene que otorgar un permiso y llevan años así, no hay apoyo gubernamental.

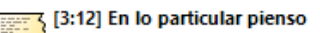
que, 5. .

En lo particular pienso que, si porque yo estoy llevando contaduría y finanzas y tenemos que utilizar las computadoras, pero restringen el uso por que otras ingenieras lo que están haciendo es reubicar los horarios.

[3:16] Si, por que en mi universidad ..

Si, por que en mi universidad no hacen las cosas a las medidas como fueron los tableros de basquetbol.

[3:4] Jugamos en una cancha
de cemen..

Fuente: Elaboración propia 
Revista Iberoamericana

de las Ciencias Sociales y

Humanísticas

ISSN: $2395-7972$

\section{Resultados y discusión}

Figura 14. Conteo de códigos. Resalta la capacidad instalada y los espacios confortables

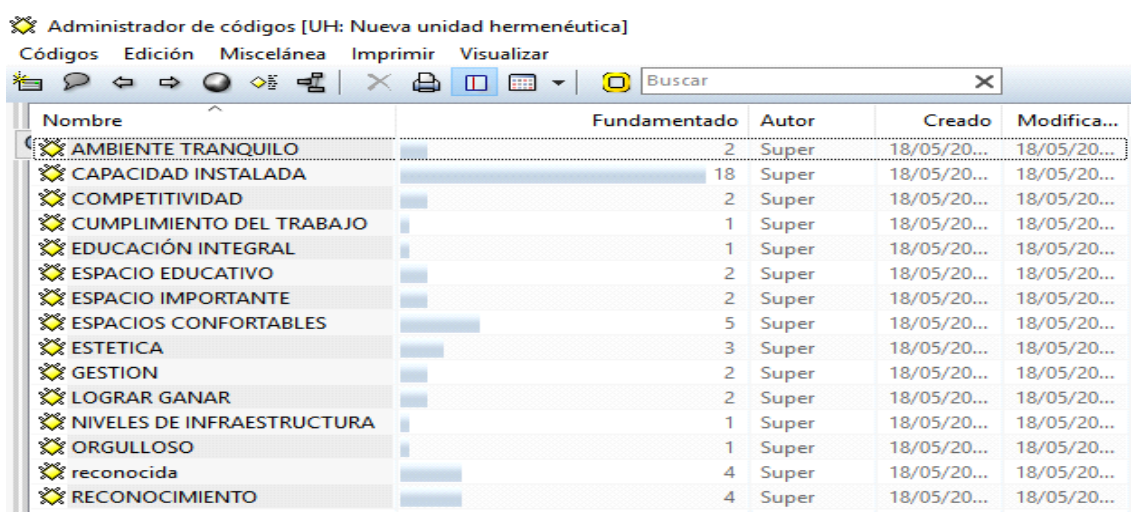

Fuente: Elaboración propia

Figura 15. Modelo que contempla las acciones a seguir

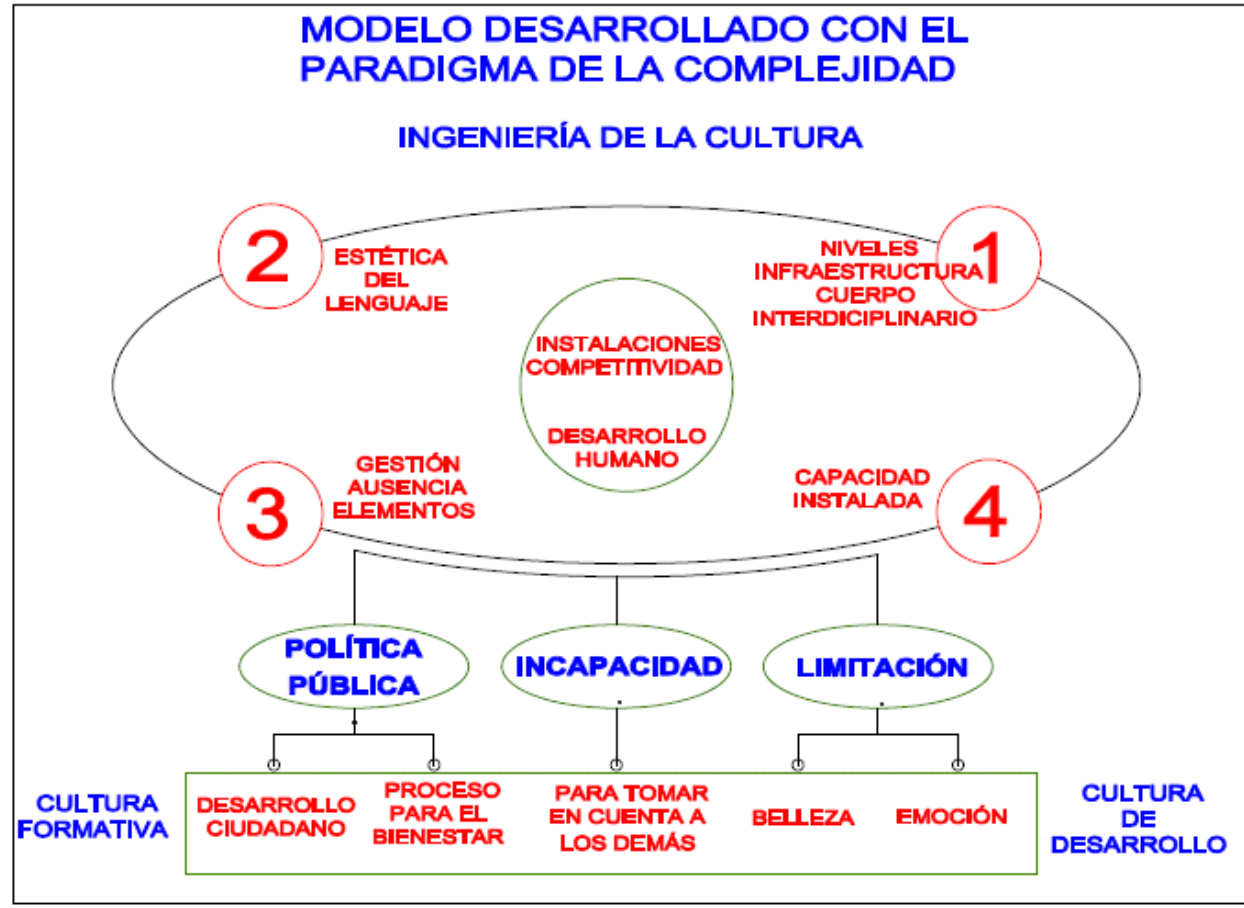

Fuente: Elaboración propia

La teoría explica y sustenta, mediante diversos autores, la necesidad de la modernidad educativa. Este documento evidencia las carencias y condiciones de espacios educativos actuales. Dichos espacios son medios por los cuales los sujetos pueden 


\section{Revista Iberoamericana \\ de las Ciencias Sociales y \\ Humanísticas}

ISSN: $2395-7972$

desarrollarse de manera integral, y también son sitios insuficientes que afectan la enseñanza y el aprendizaje.

Las entrevistas y el grupo de enfoque indican que puede mejorar el rendimiento estudiantil, la enseñanza, el aprendizaje en el aula y en el deporte, que, a su vez, se obtiene con el paradigma de la complejidad, un modelo que refleja orden, equilibrio y entendimiento de un sistema como fortaleza importante; documenta técnicas para las propuestas y mejoras en espacios educativos que inciden en un mayor incremento de la competitividad y modernidad educativa.

\section{Conclusiones}

En la investigación teórica existe una tendencia dirigida a un progreso y modernidad para la educación. El trabajo de campo documentó datos que reflejan la problemática y la necesidad de los estudiantes tienen para realizar sus actividades. Comprometiéndose a progresar, modificarse $y$ erigirse por medio de la interdisciplinariedad, debe ser el motor que conduzca a nuestro país a producir espacios escolares confortables y de calidad.

Figura 16. Reflejo del orden en los espacios educativos

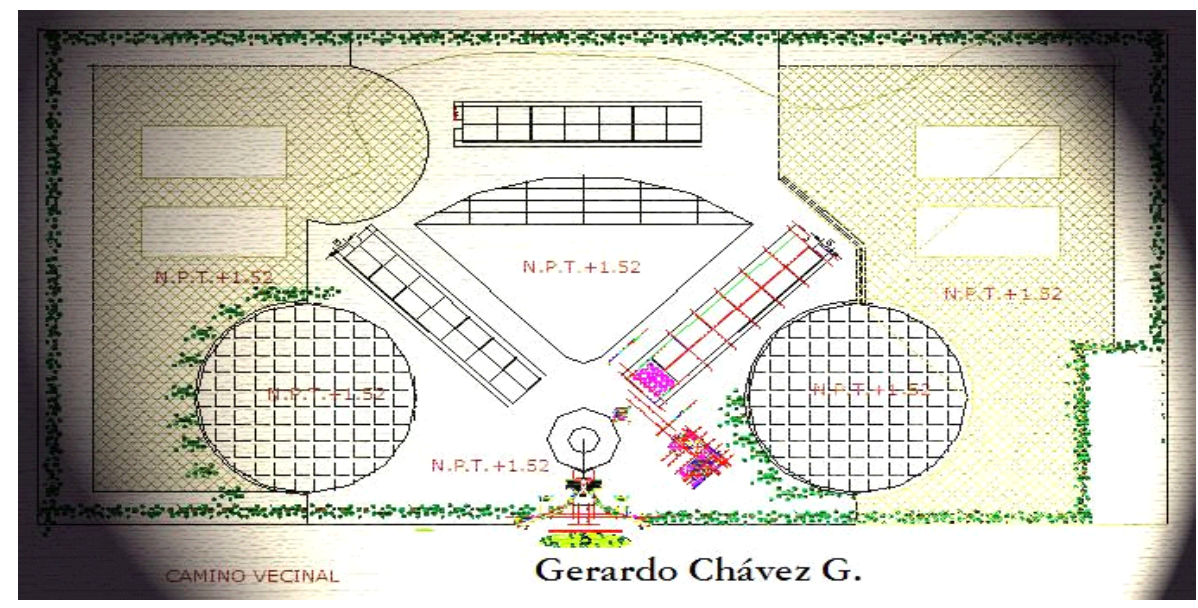

Fuente: Elaboración propia

Esta investigación invita a participar en el diseño de espacios educativos que sean armónicos, equilibrados, bellos y trascendentales para el hombre. 


\section{Revista Iberoamericana \\ de las Ciencias Sociales y \\ Humanísticas}

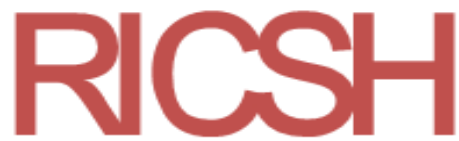

ISSN: $2395-7972$

Figura 17. Imagen utópica de las escuelas del futuro

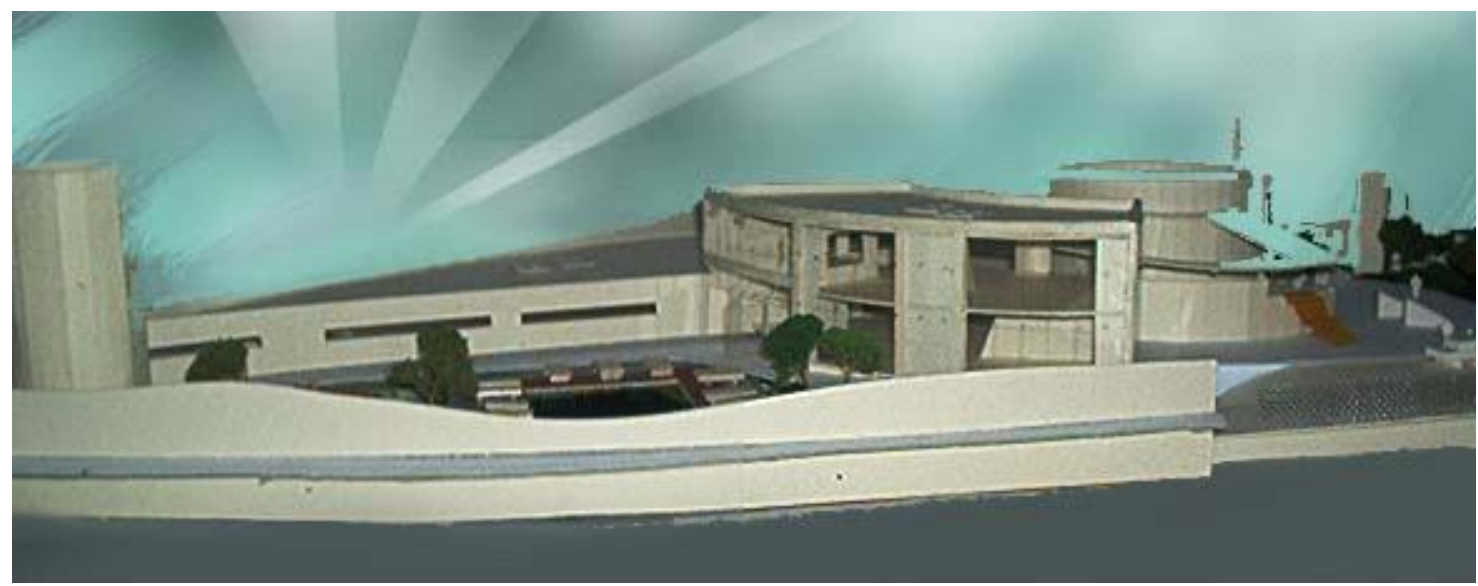

Fuente: Elaboración propia

En conclusión, se puede mejorar el rendimiento del aprendizaje con una enseñanza integral sin olvidar la importancia que tienen los espacios escolares como una esfera de superación, que define la emoción, con el orden visual, nos demuestra disciplina, por ende, el alumno aprende a concebir un bienestar que le otorga el conocimiento conjuntamente con el espacio y que va dirigido a una calidad de vida. Se prevé que el capital humano formará sus familias y se integrará como es común en la mayoría de los casos a una organización, y fomentará cambios en la cultura, promoverá espacios sustentables en la educación de diferentes niveles, con la esperanza de crear mejores trabajadores, ejecutivos y técnicos con una formación sólida que involucre un valor espacial de identidad aparte de su educación, enfocando la atención a espacios escolares ergonómicos.

\section{Agradecimientos}

Mi profundo respeto y agradecimiento a Dios, a todos los profesores, doctores, por su tiempo y dedicación en la estancia posdoctoral, así como también a mi amada familia, tías, primos, padres, esposa e hijos y compañeros de grado. Especialmente en memoria de mi padre y hermano Romualdo. 


\section{Revista Iberoamericana \\ de las Ciencias Sociales y \\ Humanísticas}

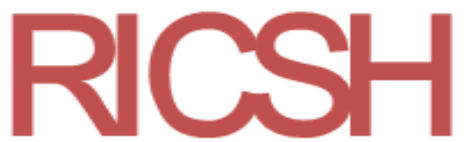

ISSN: $2395-7972$

\section{Referencias}

Abad, J. (2006). La escuela como ámbito estético según la pedagogía reggiana. Revista Aula de Infantil.

Casassus, J. (1992). Modernidad educacional y modernización educativa. Boletín Proyecto Principal de Educación en América Latina y el Caribe, (28), 33-42.

Conceptos básicos de la metodología de la investigación. (2010). Tipos de muestreo. Recuperado de http://metodologia02.blogspot.com/p/tipos-de-muestreo.html.

Coppola, P. (1997). Análisis y diseño de los espacios que habitamos. Ciudad de México, México: Editorial Pax México. Recuperado de https://www.academia.edu/40113905/ANALISIS_Y_DISENO_DE_LOS_ESPACI OS_QUE_HABITAMOS_AF_pdf.

Logroño, M. (2014). Paradigma de la complejidad. Recuperado de https://es.slideshare.net/MoisesLogroo/paradigma-de-la-complejidad-33079258.

López, O. (1998). El paradigma de la complejidad en Edgar Morín. Revista del Departamento de Ciencias: NOOS, (7), 98-115.

Lotito, F. (2009). Arquitectura. Psicología. Espacio e individuo. Revista AUS, (6), 12-17.

Ortigosa, M., González, O., Rodríguez, L., Stanfor, A., Villalobos, E., Marines, J., Perozo, M. y Castillo R. (2003). El rendimiento y la prosecución estudiantil en la escuela de arquitectura, LUZ. Aproximación a un modelo de evaluación permanente. Revista Multiciencia, 3(2).

Salmerón, H. (1989). Evaluación de la calidad de los espacios arquitectónicos escolares. (Tesis). Universidad Nacional de Educación a Distancia, España. 